Research Article

\title{
Scientific Evidence of Xuebijing Injection in the Treatment of Sepsis
}

\author{
Sa Tian $\left(\mathbb{D}\right.$, Defang Qin $\left(\mathbb{D}\right.$, Yixuan Ye $\mathbb{D}^{D}$, Huawei Yang $(\mathbb{D}$, Shuguang Chen $(\mathbb{D}$, Tao Liu $(\mathbb{D}$, \\ Luming Hu $\mathbb{D}^{\mathbb{D}}$, Huiming $\mathrm{Li} \mathbb{D}^{\mathrm{D}}$, Qian Niu $\mathbb{D}$, and Xingzhan Zhang
}

Department of Intensive Care Unit, Guangdong Provincial Hospital of Chinese Medicine, Zhuhai, China

Correspondence should be addressed to Xingzhan Zhang; 13928000908@163.com

Received 15 July 2021; Accepted 7 October 2021; Published 25 October 2021

Academic Editor: Dejan Stojkovic

Copyright (c) 2021 Sa Tian et al. This is an open access article distributed under the Creative Commons Attribution License, which permits unrestricted use, distribution, and reproduction in any medium, provided the original work is properly cited.

\begin{abstract}
Objectives. To systematically collate, appraise, and synthesize the current evidence on the Xuebijing injection (XBJI) for sepsis. Methods. Eight databases were searched for systematic reviews (SRs) or meta-analyses (MAs) on XBJI for sepsis. Assessing the Methodological Quality of Systematic Reviews-2 (AMSTAR-2), Preferred Reporting Item for Systematic Reviews and MetaAnalyses (PRISMA), and Grading of Recommendations, Assessment, Development, and Evaluation (GRADE) methods were used to assess the methodological quality, reporting quality, and evidence quality of the enrolled studies, respectively. Results. Out of the 13 studies that were included, all studies were rated critically low quality based on AMSTAR-2 results. Based on the results obtained from PRISMA, all studies were reported to be over $80 \%$, while the GRADE system yielded three outcome measures rated high-quality, 16 were of moderate quality, and the rest were of low or critically low quality. Conclusions. The combination of XBJI and Western medicine (WM) showed significant synergy for the treatment of sepsis compared to WM alone. However, this conclusion should be treated with caution since the quality of the SRs/MAs providing the evidence was relatively low.
\end{abstract}

\section{Introduction}

Sepsis is a severe complication resulting from severe infection, severe trauma, burns, surgery, and shock. This complication is quite perilous, leading to septic shock and multiple organ dysfunction syndromes [1]. Sepsis is a common cause of death in the intensive care unit [2], where it accounts for one-third to one-half of hospital deaths [3], as more than six million people die from this disease worldwide each year [4]. Despite the major advances in antibiotics and supportive therapies over the last few decades, mortality from sepsis still maintains an increasing trend [5]. At present, there is still no effective treatment for sepsis. Conventional anti-infection and supportive therapies have also shown no significant improvement in the survival rate of patients with sepsis $[6,7]$. Under these circumstances, complementary and alternative therapies have drawn attention.

Xuebijing injection (XBJI), a Chinese patent medicine, was developed by Professor Jinda Wang [8]. Professor
Wang's work was based on the Xuefu Zhuyu decoction by Wang Qingren, a famous physician in the Qing dynasty. XBJI consists of five Chinese herbs (Radix Salviae, Carthami Flos, Chuanxiong Rhizoma, Angelicae Sinensis Radix, and Paeoniae Radix Alba) that contain approximately 30 bioactive compounds, such as hydroxysafflor yellow A, danshenol, ferulic acid, paeoniflorin, senna lactone I, and more [9]. The benefits of this medicine include detoxification and toning, elimination of bacteria and viruses, supplementation of vital energy, and improved blood circulation $[9,10]$. In addition, the medicine can also inhibit the action of most inflammatory mediators and endotoxin, allowing the restoration of the immune response [11]. It was reported that XBJI can block the progression of sepsis through different mechanisms, such as antibacterial, antioxidative, and antiendotoxin $[8,11]$. Therefore, XBJI has been approved as a State Category II New Drug for the treatment of sepsis in China and has been used in clinical practice [10]. Previous studies have shown that integrated medicine can reduce mortality due to sepsis [8], but the efficacy of XBJI combined 
with Western medicine (WM) still lacks scientific evidence. This study aims to systematically collate, appraise, and synthesize scientific evidence through the presentation of an overview of these SRs/MAs.

\section{Methods}

This study was registered in the PROSPERO registry (CRD42021264569). The methods of the Cochrane handbook and some high-quality reviews were followed $[12,13]$.

2.1. Strategy for Search. A systematic search was conducted utilizing PubMed, Cochrane Library, Embase, Web of Science, China National Knowledge Infrastructure, Chongqing VIP, SinoMed, and Wanfang databases from inception to June 2021. The following medical subject headings, terms, and relevant keywords were used in this search: Xuebijing, sepsis, and systematic review. The search strategies can be found in additional file 1 .

2.2. Criteria of Inclusion and Exclusion. The studies that met the following criteria would be included for further evaluation. (1) Study type: participants enrolled in randomized controlled trials. (2) Subjects: patients diagnosed with sepsis according to internationally recognized diagnostic criteria. (3) Interventions: the experimental intervention was a combination of XBJI plus WM and the control intervention was WM alone. (4) Outcomes: one or more of the index of outcomes was present, such as 28-day mortality, acute physiology and chronic health evaluation (APACHE) II score, white blood cell, and procalcitonin (PCT). A study was excluded if it had the following factors: (1) it was a duplicate publication, (2) it was an expert comment or a conference report, (3) it did not undergo peer review, (4) the control group included XBJI, and (5) the lack of further data.

\subsection{Literature Selection and Data Extraction. Two inde-} pendent authors strictly followed the inclusion and exclusion criteria to conduct the study selection. Titles and abstracts of the literature were screened first, followed by the full text of all the initial qualified literature. The following data were extracted from each study: general information (first authors, country, and publication year), characteristics of the study (sample size and interventions), and results (outcomes and relative effect). A third author resolved any discrepancies between the two authors.

2.4. Quality Assessment. For the eligible studies that were included, two independent authors assessed the methodological quality, reporting quality, and evidence quality using the appraisal tool for systematic reviews of randomized and observational studies, Assessing the Methodological Quality of Systematic Reviews-2 (AMSTAR-2) [14], Preferred Reporting Item for Systematic Reviews and Meta-Analyses (PRISMA) [15], and Grading of Recommendations, Assessment, Development, and Evaluation (GRADE) [16], respectively. A third author resolved any discrepancies between the two authors. The items obtained from AMSTAR-2 and the checklists of PRISMA can be found in the additional file 2 and additional file 3.

\section{Results}

3.1. Results of the Literature Search. From the databases utilized, 132 articles were identified from the initial search. After 63 duplicate articles were removed, 69 were eliminated based on the title and abstract following the criteria. Then, the eligibility of the remaining 22 articles was evaluated by scanning the full text of each article. Finally, examining full text resulted in the exclusion of eight studies (Appendix file 4 ), and the remaining 14 studies [17-30] met the inclusion criteria. Flow diagram of the literature selection process is shown in Figure 1.

3.2. Basic Characteristics. The studies included were published between 2010 and 2021. Five of these reviews were published in English, while the remaining were in Chinese. The number of trials of the included reviews ranged from 11 to 49 studies, and the total number of subjects ranged from 399 to 1970 . As for the intervention, all reviews compared $\mathrm{XBJI}$ plus WM as a treatment intervention, while the control group only utilized WM. Six reviews out of 13 applied the Jadad scale for methodological quality assessment of included trials, while the remaining seven reviews used the Cochrane criteria tool. Further details of the assessment are given in Table 1.

\subsection{Quality Assessment}

3.3.1. Methodological Appraisal. The methodological quality was evaluated through AMSTAR-2. Among these studies, items $2,4,7,9,11,13$, and 15 were identified as key items. The key factors affecting the methodological quality were item 2 (no review established protocol), item 4 (11 reviews did not provide the search strategy), item 7 (no review provided a list of excluded trials), item 10 (6 reviews did not report the sources of funding), and item 16 (5 reviews did not report any potential sources of conflict of interest). Further details of this assessment are given in Table 2.

3.3.2. Quality of Reporting Appraisal. The quality of reporting was evaluated using the PRISMA guidelines, which included 7 sections and 27 items. The sections of the studies, including project title, abstract, introductions, and discussion, were comprehensively reported $(100 \%)$. In the Methods section, the protocol and registration numbers were not reported in any of the reviews (0\%), while the searches were completely reported in three reviews (21.4\%), and the additional analyses conducted in the studies were reported in 10 reviews $(78.6 \%)$. In the results section, the additional analyses were reported in 8 reviews (57.1\%). Furthermore, funding was only reported in 8 reviews (57.1\%). Further details are given in Table 3. 


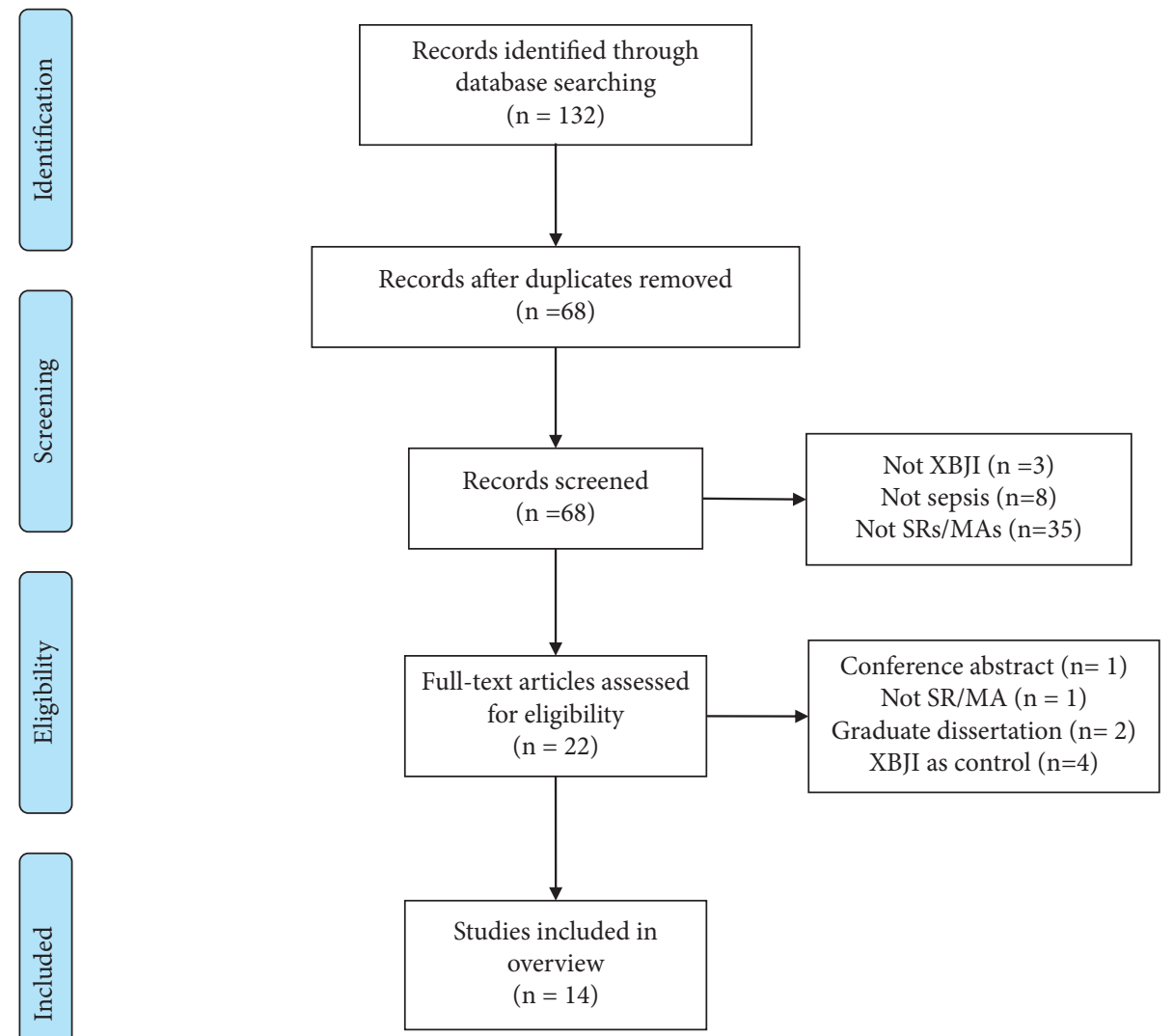

FIgURE 1: Flow diagram of the literature selection process.

3.3.3. GRADE Evidence Quality Classification. The 13 reviews included 43 outcome indicators that were related to the effectiveness of XBJI for sepsis. Three outcomes were identified as high quality, 16 were identified to be of moderate quality, 19 were identified to be of low quality, and the remaining 3 were identified to be with critically low quality. The risk of bias, inconsistency, imprecision, and publication bias were the main reasons for the decrease in quality. Further details are given in Table 4.

\subsection{Description of Efficacy}

3.4.1. Effect of the Interventions. The effects of the outcome indicators related to the effectiveness of XBJI for sepsis are given in Table 4. Twelve reviews $[17,24,26,27,29,30]$ reported the meta-analysis results of the 28 -day mortality. The results revealed that the 28 -day mortality rate of the XBJI group was lower when compared to the control group. Night reviews $[17,21,23,25,27,30]$ reported the outcomes for the APACHE II score revealed that XBJI combined with WM was superior to a single WM in improving the APACHE II score. Three reviews $[17,19,20]$ then reported the outcomes for the duration of mechanical ventilation. These results showed that the time of mechanical ventilation of XBJI combined with the WM group was shorter than the control group, while three reviews $[17,19,20]$ reported that the outcomes for the length of ICU stay showed that the XBJI plus WM group had an advantage over the WM only group in reducing the length of ICU stay. Two reviews $[18,20]$ reported the outcomes for body temperature changes, where their results revealed that XBJI accompanied with WM could lower body temperature better than the treatment with WM alone. Five reviews $[17,19,21,22,25]$ then reported the serum levels of PCT for $\mathrm{XBJI}$ in combination with WM and the control group. The results showed that the XBJI plus WM group had a lower PCT level than the control group. Furthermore, four reviews $[18,25,27,28,30]$ reported that the white blood cell count of the XBJI plus WM group was lower than the control group.

3.4.2. Safety of the Interventions. A total of five reviews $[18,19,24,25,29]$ mentioned the adverse effects of XBJI for sepsis. Wherein, no adverse effects were reported in 3 reviews [18, 24, 25]. Two reviews [19, 29] identified the following side effects, including pruritus and mild diarrhea, but no significant difference was found compared with the control group.

\section{Discussion}

The treatment of sepsis remains unsatisfactory despite the use of combined antibiotics and therapy [18]. Therefore, it is essential to identify a more effective, innovative, and 
Table 1: Basic characteristics description.

\begin{tabular}{|c|c|c|c|c|c|c|}
\hline Author, year & Country & $\begin{array}{l}\text { Sample } \\
\text { size }\end{array}$ & $\begin{array}{l}\text { Treatment } \\
\text { intervention }\end{array}$ & $\begin{array}{c}\text { Control } \\
\text { intervention }\end{array}$ & $\begin{array}{l}\text { Quality } \\
\text { assessment } \\
\text { tool }\end{array}$ & Conclusion summary \\
\hline $\begin{array}{l}\text { Chen et al. } \\
\text { [17] } 2018\end{array}$ & China & $\begin{array}{c}17 \\
(1247)\end{array}$ & XBJI + WM & WM & $\begin{array}{l}\text { Cochrane } \\
\text { criteria }\end{array}$ & $\begin{array}{l}\text { The XBJI and ulinastatin combination therapy } \\
\text { appeared to be more effective for the treatment of } \\
\text { sepsis when compared with the use of ulinastatin } \\
\text { alone. }\end{array}$ \\
\hline $\begin{array}{l}\text { Li et al. [18] } \\
2018\end{array}$ & China & $\begin{array}{c}16 \\
(1144)\end{array}$ & $\mathrm{XBJI}+\mathrm{WM}$ & WM & $\begin{array}{l}\text { Cochrane } \\
\text { criteria }\end{array}$ & $\begin{array}{l}\text { This study suggested that supplementation with } \\
\text { XBJI in addition to the conventional treatment } \\
\text { appeared to be more effective for the treatment of } \\
\text { sepsis as compared to the conventional treatment } \\
\text { alone. }\end{array}$ \\
\hline $\begin{array}{l}\text { Xiao et al. } \\
\text { [19] } 2018\end{array}$ & China & $\begin{array}{c}16 \\
(1335)\end{array}$ & XBJI + WM & WM & $\begin{array}{l}\text { Cochrane } \\
\text { criteria }\end{array}$ & $\begin{array}{l}\text { The combination therapy appeared to be more } \\
\text { effective for the treatment of sepsis compared to the } \\
\text { conventional treatment alone. It was also observed } \\
\text { that the risk of adverse events did not increase. }\end{array}$ \\
\hline $\begin{array}{l}\text { Zheng et al. } \\
\text { [20] } 2018\end{array}$ & China & $\begin{array}{c}16 \\
(1192)\end{array}$ & XBJI + WM & WM & $\begin{array}{l}\text { Cochrane } \\
\text { criteria }\end{array}$ & $\begin{array}{l}\text { Our results found that XBJI when combined with } \\
\text { ulinastatin was superior to both routine therapies } \\
\text { and the single administration of either ulinastatin } \\
\text { or XBJI. }\end{array}$ \\
\hline $\begin{array}{l}\text { Xiao et al. } \\
\text { [21] } 2017\end{array}$ & China & $49(1801)$ & XBJI + WM & WM & Jadad & $\begin{array}{l}\text { The combination therapy appeared to be more } \\
\text { effective for the treatment of sepsis when compared } \\
\text { to conventional treatment alone. }\end{array}$ \\
\hline $\begin{array}{l}\text { Liu et al. } \\
\text { [22] } 2021\end{array}$ & China & $\begin{array}{c}16 \\
(1423)\end{array}$ & XBJI + WM & WM & Jadad & $\begin{array}{l}\text { This study suggested that supplementation with } \\
\text { XBJI in addition to the conventional treatment } \\
\text { appeared to be more effective for the treatment of } \\
\text { sepsis as compared to conventional treatment } \\
\text { alone. }\end{array}$ \\
\hline $\begin{array}{l}\text { Zhang et al. } \\
\text { [23] } 2021\end{array}$ & China & $15(930)$ & $\mathrm{XBJI}+\mathrm{WM}$ & WM & $\begin{array}{l}\text { Cochrane } \\
\text { criteria }\end{array}$ & $\begin{array}{l}\text { The utilization of XBJI has a certain effect on the } \\
\text { improvement of the inflammatory response and } \\
\text { increased level of platelets. }\end{array}$ \\
\hline $\begin{array}{l}\text { Zhou et al. } \\
\text { [24] } 2016\end{array}$ & China & $8(399)$ & XBJI + WM & WM & Jadad & $\begin{array}{l}\text { The homogeneity of the reduced mortality rate and } \\
\text { the available evidence was sufficient to support the } \\
\text { use of XBJI as adjunctive therapy for sepsis. }\end{array}$ \\
\hline $\begin{array}{l}\text { Li et al. [25] } \\
2016\end{array}$ & China & $11(803)$ & XBJI + WM & WM & $\begin{array}{l}\text { Cochrane } \\
\text { criteria }\end{array}$ & $\begin{array}{l}\text { Clinical evidence showed that the addition of XBJI } \\
\text { to the conventional treatment could improve the } \\
\text { clinical efficacy in the treatment of sepsis. }\end{array}$ \\
\hline $\begin{array}{l}\text { Xu et al. [26] } \\
2014\end{array}$ & China & $\begin{array}{c}18 \\
(1172)\end{array}$ & XBJI + WM & WM & Jadad & $\begin{array}{l}\text { The combined use of XBJI based on conventional } \\
\text { treatment could improve the survival rate of } \\
\text { patients with sepsis. }\end{array}$ \\
\hline $\begin{array}{l}\text { Li et al. [27] } \\
2013\end{array}$ & China & $\begin{array}{c}13 \\
(1280)\end{array}$ & XBJI + WM & WM & Jadad & $\begin{array}{l}\text { XBJI had a certain effect in improving the } \\
\text { inflammatory response and coagulation function in } \\
\text { patients with sepsis. These effects reduced mortality } \\
\text { and improved the APACHE II scores. }\end{array}$ \\
\hline $\begin{array}{l}\text { Sun et al. } \\
\text { [28] } 2012\end{array}$ & China & $\begin{array}{c}18 \\
(1080)\end{array}$ & XBJI + WM & WM & Jadad & $\begin{array}{l}\text { The existing results showed that the application of } \\
\text { XBJI in the treatment of sepsis could significantly } \\
\text { reduce the white blood cell count in the plasma of } \\
\text { patients. }\end{array}$ \\
\hline $\begin{array}{l}\text { Hu et al, } \\
\text { [29] } 2010\end{array}$ & China & $\begin{array}{c}25 \\
(1970)\end{array}$ & XBJI + WM & WM & $\begin{array}{l}\text { Cochrane } \\
\text { criteria }\end{array}$ & $\begin{array}{l}\text { The evidence available showed that XBJI might } \\
\text { decrease mortality, ineffectiveness, incidence of } \\
\text { complication, and average hospital stay. It could } \\
\text { also reduce the APACHE II score in patients with } \\
\text { sepsis. }\end{array}$ \\
\hline $\begin{array}{l}\text { Wu et al. } \\
\text { [30] } 2020\end{array}$ & China & $14(938)$ & XBJI + WM & WM & $\begin{array}{l}\text { Cochrane } \\
\text { criteria }\end{array}$ & $\begin{array}{l}\text { XBJI can improve the clinical symptoms, } \\
\text { significantly reduce the mortality, and has a high } \\
\text { clinical application value. }\end{array}$ \\
\hline
\end{tabular}

adjunctive medicine for clinical application [17]. XBJI has been widely used for sepsis in clinical practices in China, wherein pharmacological experiments have demonstrated that it may be a promising treatment for sepsis. As the number of SRs/MAs regarding XBJI for sepsis has increased, scientific evidence for evidence-based medicine is still weak. Hence, we conducted this study to collate, appraise, and synthesize the evidence on XBJI systematically. 
TABLE 2: Result of methodological quality.

\begin{tabular}{|c|c|c|c|c|c|c|c|c|c|c|c|c|c|c|c|c|c|}
\hline \multirow{2}{*}{ Reviews } & \multicolumn{16}{|c|}{ AMSTAR-2 } & \multirow{2}{*}{ Quality } \\
\hline & I1 & $\mathrm{I} 2$ & $\mathrm{I} 3$ & $\mathrm{I} 4$ & I5 & I6 & I7 & I8 & I9 & $\mathrm{I} 10$ & I11 & $\mathrm{I} 12$ & $\mathrm{I} 13$ & $\mathrm{I} 14$ & $\mathrm{I} 15$ & I16 & \\
\hline Chen et al. [17] 2018 & $\mathrm{Y}$ & PY & $\mathrm{Y}$ & $\mathrm{Y}$ & $\mathrm{Y}$ & $\mathrm{Y}$ & $\mathrm{N}$ & $\mathrm{Y}$ & $\mathrm{Y}$ & $\mathrm{Y}$ & $\mathrm{Y}$ & $\mathrm{Y}$ & $\mathrm{Y}$ & $\mathrm{Y}$ & $\mathrm{Y}$ & $\mathrm{Y}$ & $\mathrm{CL}$ \\
\hline Li et al. [18] 2018 & $\mathrm{Y}$ & PY & $\mathrm{Y}$ & $\mathrm{Y}$ & $\mathrm{Y}$ & $\mathrm{Y}$ & $\mathrm{N}$ & $\mathrm{Y}$ & $\mathrm{Y}$ & $\mathrm{Y}$ & $\mathrm{Y}$ & $\mathrm{Y}$ & $\mathrm{Y}$ & $\mathrm{Y}$ & $\mathrm{Y}$ & $\mathrm{Y}$ & $\mathrm{CL}$ \\
\hline Xiao et al. [19] 2018 & $\mathrm{Y}$ & PY & $\mathrm{Y}$ & $\mathrm{Y}$ & $\mathrm{Y}$ & $\mathrm{Y}$ & $\mathrm{N}$ & $\mathrm{Y}$ & $\mathrm{Y}$ & $\mathrm{Y}$ & $\mathrm{Y}$ & $\mathrm{Y}$ & $\mathrm{Y}$ & $\mathrm{Y}$ & $\mathrm{Y}$ & $\mathrm{Y}$ & $\mathrm{CL}$ \\
\hline Zheng et al. [20] 2018 & $\mathrm{Y}$ & PY & $\mathrm{Y}$ & PY & $\mathrm{Y}$ & $\mathrm{Y}$ & $\mathrm{N}$ & $\mathrm{Y}$ & $\mathrm{Y}$ & $\mathrm{Y}$ & $\mathrm{Y}$ & $\mathrm{Y}$ & $\mathrm{Y}$ & $\mathrm{Y}$ & $\mathrm{Y}$ & $\mathrm{Y}$ & $\overline{C L}$ \\
\hline Xiao et al. [21] 2017 & $\mathrm{Y}$ & PY & $\mathrm{Y}$ & PY & $\mathrm{Y}$ & $\mathrm{Y}$ & $\mathrm{N}$ & $\mathrm{Y}$ & $\mathrm{Y}$ & $\mathrm{Y}$ & $\mathrm{Y}$ & $\mathrm{Y}$ & $\mathrm{Y}$ & $\mathrm{Y}$ & $\mathrm{Y}$ & $\mathrm{Y}$ & $\mathrm{CL}$ \\
\hline Liu et al. [22] 2021 & $\mathrm{Y}$ & PY & $\mathrm{Y}$ & PY & $\mathrm{Y}$ & $\mathrm{Y}$ & $\mathrm{N}$ & $\mathrm{Y}$ & $\mathrm{Y}$ & $\mathrm{Y}$ & $\mathrm{Y}$ & $\mathrm{Y}$ & $\mathrm{Y}$ & $\mathrm{Y}$ & $\mathrm{Y}$ & $\mathrm{Y}$ & $\mathrm{CL}$ \\
\hline Zhang et al. [23] 2021 & $\mathrm{Y}$ & PY & $\mathrm{Y}$ & PY & $\mathrm{Y}$ & $\mathrm{Y}$ & $\mathrm{N}$ & $\mathrm{Y}$ & $\mathrm{Y}$ & $\mathrm{Y}$ & $\mathrm{Y}$ & $\mathrm{Y}$ & $\mathrm{Y}$ & $\mathrm{Y}$ & $\mathrm{Y}$ & $\mathrm{Y}$ & $\mathrm{CL}$ \\
\hline Zhou et al. [24] 2016 & $\mathrm{Y}$ & PY & $\mathrm{Y}$ & PY & $\mathrm{Y}$ & $\mathrm{Y}$ & $\mathrm{N}$ & $\mathrm{Y}$ & $\mathrm{Y}$ & $\mathrm{Y}$ & $\mathrm{Y}$ & $\mathrm{Y}$ & $\mathrm{Y}$ & $\mathrm{Y}$ & $\mathrm{Y}$ & $\mathrm{Y}$ & $\mathrm{CL}$ \\
\hline Li et al. [25] 2016 & $\mathrm{Y}$ & PY & $\mathrm{Y}$ & PY & $\mathrm{Y}$ & $\mathrm{Y}$ & $\mathrm{N}$ & $\mathrm{Y}$ & $\mathrm{Y}$ & $\mathrm{N}$ & $\mathrm{Y}$ & $\mathrm{Y}$ & $\mathrm{Y}$ & $\mathrm{Y}$ & $\mathrm{Y}$ & $\mathrm{N}$ & CL \\
\hline Xu et al. [26] 2014 & $\mathrm{Y}$ & PY & $\mathrm{Y}$ & PY & $\mathrm{Y}$ & $\mathrm{Y}$ & $\mathrm{N}$ & $\mathrm{Y}$ & $\mathrm{Y}$ & $\mathrm{N}$ & $\mathrm{Y}$ & $\mathrm{Y}$ & $\mathrm{Y}$ & $\mathrm{Y}$ & $\mathrm{Y}$ & $\mathrm{N}$ & $\mathrm{CL}$ \\
\hline Li et al. [27] 2013 & $\mathrm{Y}$ & PY & $\mathrm{Y}$ & PY & $\mathrm{Y}$ & $\mathrm{Y}$ & $\mathrm{N}$ & $\mathrm{Y}$ & $\mathrm{Y}$ & $\mathrm{N}$ & $\mathrm{Y}$ & $\mathrm{Y}$ & $\mathrm{Y}$ & $\mathrm{Y}$ & $\mathrm{Y}$ & $\mathrm{N}$ & $\mathrm{CL}$ \\
\hline Sun et al. [28] 2012 & $\mathrm{Y}$ & PY & $\mathrm{Y}$ & PY & $\mathrm{Y}$ & $\mathrm{Y}$ & $\mathrm{N}$ & $\mathrm{Y}$ & $\mathrm{Y}$ & $\mathrm{N}$ & $\mathrm{Y}$ & $\mathrm{Y}$ & $\mathrm{Y}$ & $\mathrm{Y}$ & $\mathrm{Y}$ & $\mathrm{N}$ & $\mathrm{CL}$ \\
\hline Hu et al. [29] 2010 & $\mathrm{Y}$ & PY & $\mathrm{Y}$ & PY & $\mathrm{Y}$ & $\mathrm{Y}$ & $\mathrm{N}$ & $\mathrm{Y}$ & $\mathrm{Y}$ & $\mathrm{N}$ & $\mathrm{Y}$ & $\mathrm{Y}$ & $\mathrm{Y}$ & $\mathrm{Y}$ & $\mathrm{Y}$ & $\mathrm{N}$ & $\mathrm{CL}$ \\
\hline Wu et al. [30] 2020 & $\mathrm{Y}$ & PY & $\mathrm{Y}$ & PY & $\mathrm{Y}$ & $\mathrm{Y}$ & $\mathrm{N}$ & $\mathrm{Y}$ & $\mathrm{Y}$ & $\mathrm{N}$ & $\mathrm{Y}$ & $\mathrm{Y}$ & $\mathrm{Y}$ & $\mathrm{Y}$ & $\mathrm{Y}$ & $\mathrm{Y}$ & $\mathrm{CL}$ \\
\hline
\end{tabular}

TABLE 3: Result of reporting quality.

\begin{tabular}{|c|c|c|c|c|c|c|c|c|c|c|c|c|c|c|c|}
\hline Items & $\begin{array}{c}\text { Chen, } \\
2018\end{array}$ & $\begin{array}{c}\mathrm{Li}, \\
2018 \\
\end{array}$ & $\begin{array}{c}\text { Xiao, } \\
2018 \\
\end{array}$ & $\begin{array}{c}\text { Zheng, } \\
2018\end{array}$ & $\begin{array}{l}\text { Xiao, } \\
2017 \\
\end{array}$ & $\begin{array}{l}\text { Liu, } \\
2021\end{array}$ & $\begin{array}{c}\text { Zhang, } \\
2021 \\
\end{array}$ & $\begin{array}{c}\text { Zhou, } \\
2016\end{array}$ & $\begin{array}{c}\mathrm{Li}, \\
2016\end{array}$ & $\begin{array}{c}\mathrm{Xu}, \\
2014 \\
\end{array}$ & $\begin{array}{c}\mathrm{Li}, \\
2014\end{array}$ & $\begin{array}{l}\text { Sun, } \\
2012\end{array}$ & $\begin{array}{l}\mathrm{Hu}, \\
2010\end{array}$ & $\begin{array}{l}\mathrm{Wu}, \\
2020\end{array}$ & $\begin{array}{c}\text { Compliance } \\
(\%)\end{array}$ \\
\hline \# 1 & $\mathrm{Y}$ & $\mathrm{Y}$ & $\mathrm{Y}$ & $\mathrm{Y}$ & $\mathrm{Y}$ & $\mathrm{Y}$ & $\mathrm{Y}$ & $\mathrm{Y}$ & $\mathrm{Y}$ & $\mathrm{Y}$ & $\mathrm{Y}$ & $\mathrm{Y}$ & $\mathrm{Y}$ & $\mathrm{Y}$ & 100 \\
\hline$\# 2$ & $\mathrm{Y}$ & $\mathrm{Y}$ & $\mathrm{Y}$ & $\mathrm{Y}$ & $\mathrm{Y}$ & $\mathrm{Y}$ & $\mathrm{Y}$ & $\mathrm{Y}$ & $\mathrm{Y}$ & $\mathrm{Y}$ & $\mathrm{Y}$ & $\mathrm{Y}$ & $\mathrm{Y}$ & $\mathrm{Y}$ & 100 \\
\hline$\# 3$ & $\mathrm{Y}$ & $\mathrm{Y}$ & $\mathrm{Y}$ & $\mathrm{Y}$ & $\mathrm{Y}$ & $\mathrm{Y}$ & $\mathrm{Y}$ & $\mathrm{Y}$ & $\mathrm{Y}$ & $\mathrm{Y}$ & $\mathrm{Y}$ & $\mathrm{Y}$ & $\mathrm{Y}$ & $\mathrm{Y}$ & 100 \\
\hline$\# 4$ & $\mathrm{Y}$ & $\mathrm{Y}$ & $\mathrm{Y}$ & $\mathrm{Y}$ & $\mathrm{Y}$ & $\mathrm{Y}$ & $\mathrm{Y}$ & $\mathrm{Y}$ & $\mathrm{Y}$ & $\mathrm{Y}$ & $\mathrm{Y}$ & $\mathrm{Y}$ & $\mathrm{Y}$ & $\mathrm{Y}$ & 100 \\
\hline$\# 5$ & $\mathrm{~N}$ & $\mathrm{~N}$ & $\mathrm{~N}$ & $\mathrm{~N}$ & $\mathrm{~N}$ & $\mathrm{~N}$ & $\mathrm{~N}$ & $\mathrm{~N}$ & $\mathrm{~N}$ & $\mathrm{~N}$ & $\mathrm{~N}$ & $\mathrm{~N}$ & $\mathrm{~N}$ & $\mathrm{~N}$ & 0 \\
\hline$\# 6$ & $\mathrm{Y}$ & $\mathrm{Y}$ & $\mathrm{Y}$ & $\mathrm{Y}$ & $\mathrm{Y}$ & $\mathrm{Y}$ & $\mathrm{Y}$ & $\mathrm{Y}$ & $\mathrm{Y}$ & $\mathrm{Y}$ & $\mathrm{Y}$ & $\mathrm{Y}$ & $\mathrm{Y}$ & $\mathrm{Y}$ & 100 \\
\hline$\# 7$ & $\mathrm{Y}$ & $\mathrm{Y}$ & $\mathrm{Y}$ & $\mathrm{Y}$ & $\mathrm{Y}$ & $\mathrm{Y}$ & $\mathrm{Y}$ & $\mathrm{Y}$ & $\mathrm{Y}$ & $\mathrm{Y}$ & $\mathrm{Y}$ & $\mathrm{Y}$ & $\mathrm{Y}$ & $\mathrm{Y}$ & 100 \\
\hline$\# 8$ & $\mathrm{Y}$ & $\mathrm{Y}$ & $\mathrm{Y}$ & PY & PY & PY & PY & PY & PY & PY & PY & PY & PY & PY & 21.4 \\
\hline \# 9 & $\mathrm{Y}$ & $\mathrm{Y}$ & $\mathrm{Y}$ & $\mathrm{Y}$ & $\mathrm{Y}$ & $\mathrm{Y}$ & $\mathrm{Y}$ & $\mathrm{Y}$ & $\mathrm{Y}$ & $\mathrm{Y}$ & $\mathrm{Y}$ & $\mathrm{Y}$ & $\mathrm{Y}$ & $\mathrm{Y}$ & 100 \\
\hline$\# 10$ & $\mathrm{Y}$ & $\mathrm{Y}$ & $\mathrm{Y}$ & $\mathrm{Y}$ & $\mathrm{Y}$ & $\mathrm{Y}$ & $\mathrm{Y}$ & $\mathrm{Y}$ & $\mathrm{Y}$ & $\mathrm{Y}$ & $\mathrm{Y}$ & $\mathrm{Y}$ & $\mathrm{Y}$ & $\mathrm{Y}$ & 100 \\
\hline$\# 11$ & $\mathrm{Y}$ & $\mathrm{Y}$ & $\mathrm{Y}$ & $\mathrm{Y}$ & $\mathrm{Y}$ & $\mathrm{Y}$ & $\mathrm{Y}$ & $\mathrm{Y}$ & $\mathrm{Y}$ & $\mathrm{Y}$ & $\mathrm{Y}$ & $\mathrm{Y}$ & $\mathrm{Y}$ & $\mathrm{Y}$ & 100 \\
\hline$\# 12$ & $\mathrm{Y}$ & $\mathrm{Y}$ & $\mathrm{Y}$ & $\mathrm{Y}$ & $\mathrm{Y}$ & $\mathrm{Y}$ & $\mathrm{Y}$ & $\mathrm{Y}$ & $\mathrm{Y}$ & $\mathrm{Y}$ & $\mathrm{Y}$ & $\mathrm{Y}$ & $\mathrm{Y}$ & $\mathrm{Y}$ & 100 \\
\hline$\# 13$ & $\mathrm{Y}$ & $\mathrm{Y}$ & $\mathrm{Y}$ & $\mathrm{Y}$ & $\mathrm{Y}$ & $\mathrm{Y}$ & $\mathrm{Y}$ & $\mathrm{Y}$ & $\mathrm{Y}$ & $\mathrm{Y}$ & $\mathrm{Y}$ & $\mathrm{Y}$ & $\mathrm{Y}$ & $\mathrm{Y}$ & 100 \\
\hline \# 14 & $\mathrm{Y}$ & $\mathrm{Y}$ & $\mathrm{Y}$ & $\mathrm{Y}$ & $\mathrm{Y}$ & $\mathrm{Y}$ & $\mathrm{Y}$ & $\mathrm{Y}$ & $\mathrm{Y}$ & $\mathrm{Y}$ & $\mathrm{Y}$ & $\mathrm{Y}$ & $\mathrm{Y}$ & $\mathrm{Y}$ & 100 \\
\hline$\# 15$ & $\mathrm{Y}$ & $\mathrm{Y}$ & $\mathrm{Y}$ & $\mathrm{Y}$ & $\mathrm{Y}$ & $\mathrm{Y}$ & $\mathrm{Y}$ & $\mathrm{Y}$ & $\mathrm{Y}$ & $\mathrm{Y}$ & $\mathrm{Y}$ & $\mathrm{Y}$ & $\mathrm{Y}$ & $\mathrm{Y}$ & 100 \\
\hline$\# 16$ & $\mathrm{Y}$ & $\mathrm{Y}$ & $\mathrm{Y}$ & $\mathrm{Y}$ & $\mathrm{Y}$ & $\mathrm{N}$ & $\mathrm{Y}$ & $\mathrm{Y}$ & $\mathrm{N}$ & $\mathrm{Y}$ & $\mathrm{Y}$ & $\mathrm{N}$ & $\mathrm{Y}$ & $\mathrm{Y}$ & 78.6 \\
\hline$\# 17$ & $\mathrm{Y}$ & $\mathrm{Y}$ & $\mathrm{Y}$ & $\mathrm{Y}$ & $\mathrm{Y}$ & $\mathrm{Y}$ & $\mathrm{Y}$ & $\mathrm{Y}$ & $\mathrm{Y}$ & $\mathrm{Y}$ & $\mathrm{Y}$ & $\mathrm{Y}$ & $\mathrm{Y}$ & $\mathrm{Y}$ & 100 \\
\hline$\# 18$ & $\mathrm{Y}$ & $\mathrm{Y}$ & $\mathrm{Y}$ & $\mathrm{Y}$ & $\mathrm{Y}$ & $\mathrm{Y}$ & $\mathrm{Y}$ & $\mathrm{Y}$ & $\mathrm{Y}$ & $\mathrm{Y}$ & $\mathrm{Y}$ & $\mathrm{Y}$ & $\mathrm{Y}$ & $\mathrm{Y}$ & 100 \\
\hline$\# 19$ & $\mathrm{Y}$ & $\mathrm{Y}$ & $\mathrm{Y}$ & $\mathrm{Y}$ & $\mathrm{Y}$ & $\mathrm{Y}$ & $\mathrm{Y}$ & $\mathrm{Y}$ & $\mathrm{Y}$ & $\mathrm{Y}$ & $\mathrm{Y}$ & $\mathrm{Y}$ & $\mathrm{Y}$ & $\mathrm{Y}$ & 100 \\
\hline$\# 20$ & $\mathrm{Y}$ & $\mathrm{Y}$ & $\mathrm{Y}$ & $\mathrm{Y}$ & $\mathrm{Y}$ & $\mathrm{Y}$ & $\mathrm{Y}$ & $\mathrm{Y}$ & $\mathrm{Y}$ & $\mathrm{Y}$ & $\mathrm{Y}$ & $\mathrm{Y}$ & $\mathrm{Y}$ & $\mathrm{Y}$ & 100 \\
\hline \# 21 & $\mathrm{Y}$ & $\mathrm{Y}$ & $\mathrm{Y}$ & $\mathrm{Y}$ & $\mathrm{Y}$ & $\mathrm{Y}$ & $\mathrm{Y}$ & $\mathrm{Y}$ & $\mathrm{Y}$ & $\mathrm{Y}$ & $\mathrm{Y}$ & $\mathrm{Y}$ & $\mathrm{Y}$ & $\mathrm{Y}$ & 100 \\
\hline$\# 22$ & $\mathrm{Y}$ & $\mathrm{Y}$ & $\mathrm{Y}$ & $\mathrm{Y}$ & $\mathrm{Y}$ & $\mathrm{Y}$ & $\mathrm{Y}$ & $\mathrm{Y}$ & $\mathrm{Y}$ & $\mathrm{Y}$ & $\mathrm{Y}$ & $\mathrm{Y}$ & $\mathrm{Y}$ & $\mathrm{Y}$ & 100 \\
\hline$\# 23$ & $\mathrm{Y}$ & $\mathrm{Y}$ & $\mathrm{Y}$ & $\mathrm{Y}$ & $\mathrm{Y}$ & $\mathrm{N}$ & $\mathrm{N}$ & $\mathrm{Y}$ & $\mathrm{N}$ & $\mathrm{Y}$ & $\mathrm{Y}$ & $\mathrm{N}$ & $\mathrm{N}$ & $\mathrm{N}$ & 57.1 \\
\hline$\# 24$ & $\mathrm{Y}$ & $\mathrm{Y}$ & $\mathrm{Y}$ & $\mathrm{Y}$ & $\mathrm{Y}$ & $\mathrm{Y}$ & $\mathrm{Y}$ & $\mathrm{Y}$ & $\mathrm{Y}$ & $\mathrm{Y}$ & $\mathrm{Y}$ & $\mathrm{Y}$ & $\mathrm{Y}$ & $\mathrm{Y}$ & 100 \\
\hline$\# 25$ & $\mathrm{Y}$ & $\mathrm{Y}$ & $\mathrm{Y}$ & $\mathrm{Y}$ & $\mathrm{Y}$ & $\mathrm{Y}$ & $\mathrm{Y}$ & $\mathrm{Y}$ & $\mathrm{Y}$ & $\mathrm{Y}$ & $\mathrm{Y}$ & $\mathrm{Y}$ & $\mathrm{Y}$ & $\mathrm{Y}$ & 100 \\
\hline$\# 26$ & $\mathrm{Y}$ & $\mathrm{Y}$ & $\mathrm{Y}$ & $\mathrm{Y}$ & $\mathrm{Y}$ & $\mathrm{Y}$ & $\mathrm{Y}$ & $\mathrm{Y}$ & $\mathrm{Y}$ & $\mathrm{Y}$ & $\mathrm{Y}$ & $\mathrm{Y}$ & $\mathrm{Y}$ & $\mathrm{Y}$ & 100 \\
\hline$\# 27$ & $\mathrm{Y}$ & $\mathrm{Y}$ & $\mathrm{Y}$ & $\mathrm{Y}$ & $\mathrm{Y}$ & $\mathrm{Y}$ & $\mathrm{Y}$ & $\mathrm{Y}$ & $\mathrm{N}$ & $\mathrm{N}$ & $\mathrm{N}$ & $\mathrm{N}$ & $\mathrm{N}$ & $\mathrm{N}$ & 57.1 \\
\hline
\end{tabular}

This overview summarized the scientific evidence on the effectiveness and safety of XBJI for sepsis by evaluating the methodological quality, reporting quality, and evidence quality of SRs/MAs. The current evidence indicated that subjects treated using the combination of XBJI and WM showed a significant reduction in the 28-day mortality, APACHE II score, duration of mechanical ventilation, length of ICU stays, body temperature, serum levels of PCT, and white blood cell count as compared to those treated with WM alone. However, this conclusion must be considered with caution, given the limitations of the study. According to the results of AMSTAR-2, all reviews failed to meet the key item of I2 (established protocol) and I7 (provided the list of excluded trials), which may contribute to the possibility of risk of bias and undermine the reliability of the conclusions. Then, according to the results of PRISMA, I5 (protocol and registration protocol and registration), I8 (search), I16 (additional analyses), I23 (additional analyses), and I27 (funding) were not reported adequately. This reasoning may increase the risk of bias and affect the rigor of SRs/MAs. 
TABLE 4: Results of evidence quality.

\begin{tabular}{|c|c|c|c|c|c|c|c|c|c|}
\hline \multirow[b]{2}{*}{ Review } & \multirow[b]{2}{*}{ Outcomes } & \multicolumn{6}{|c|}{ Certainty assessment } & \multirow{2}{*}{$\begin{array}{l}\text { Relative effect } \\
\quad(95 \% \mathrm{CI})\end{array}$} & \multirow[b]{2}{*}{ Quality } \\
\hline & & Design & Limitations & Inconsistency & Indirectness & Imprecision & $\begin{array}{c}\text { Publication } \\
\text { bias }\end{array}$ & & \\
\hline \multirow{5}{*}{$\begin{array}{l}\text { Chen } \\
\text { et al. [17] } \\
2018\end{array}$} & $\begin{array}{l}28 \text { days } \\
\text { mortality }\end{array}$ & Rct & No & No & No & No & No & $\begin{array}{c}\text { RR } 0.54 \\
(0.39,0.73)\end{array}$ & $\begin{array}{l}\oplus \oplus \oplus \oplus \oplus \\
\text { high }\end{array}$ \\
\hline & $\begin{array}{l}\text { Duration of } \\
\text { mechanical } \\
\text { ventilation }\end{array}$ & Rct & No & No & No & No & No & $\begin{array}{c}\text { SMD }-1.13 \\
(-1.30,-0.95)\end{array}$ & $\begin{array}{c}\oplus \oplus \oplus \oplus \oplus \\
\text { high }\end{array}$ \\
\hline & $\begin{array}{c}\text { Length of ICU } \\
\text { stay }\end{array}$ & Rct & No & No & No & No & No & $\begin{array}{l}\text { SMD }-0.84 \\
(-1.00,-0.67)\end{array}$ & $\begin{array}{l}\oplus \oplus \oplus \oplus \oplus \\
\text { high }\end{array}$ \\
\hline & $\begin{array}{l}\text { APACHE II } \\
\text { score }\end{array}$ & Rct & No & Serious & No & No & No & $\begin{array}{l}\text { SMD }-1.09 \\
(-1.49,-0.69)\end{array}$ & $\begin{array}{l}\oplus \oplus \oplus \oplus \bigcirc \\
\text { moderate }\end{array}$ \\
\hline & $\begin{array}{c}\text { Serum levels of } \\
\text { PCT }\end{array}$ & Rct & Serious & Serious & No & No & No & $\begin{array}{c}\text { SMD }-1.61 \\
(-2.23,-0.98) \\
\end{array}$ & $\begin{array}{c}\oplus \oplus \oplus \bigcirc \bigcirc \\
\text { low } \\
\end{array}$ \\
\hline \multirow{4}{*}{$\begin{array}{l}\text { Li et al. } \\
\text { [18] } 2018\end{array}$} & $\begin{array}{l}28 \text { days } \\
\text { mortality }\end{array}$ & Rct & Serious & No & No & No & No & $\begin{array}{c}\text { RR } 0.62(0.51, \\
0.76)\end{array}$ & $\begin{array}{l}\oplus \oplus \oplus \oplus \bigcirc \\
\text { moderate }\end{array}$ \\
\hline & $\begin{array}{l}\text { APACHE II } \\
\text { score }\end{array}$ & Rct & Serious & Serious & No & No & No & $\begin{array}{c}\mathrm{MD}=-3.51 \\
(-4.49,-2.54)\end{array}$ & $\begin{array}{c}\oplus \oplus \oplus \bigcirc \bigcirc \\
\text { low }\end{array}$ \\
\hline & $\begin{array}{l}\text { White blood } \\
\text { count }\end{array}$ & Rct & Serious & Serious & No & Serious & Serious & $\begin{array}{c}\mathrm{MD}=-8.00 \\
(-10.18,-5.82)\end{array}$ & $\begin{array}{l}\oplus \bigcirc \bigcirc \bigcirc \bigcirc \\
\text { very low }\end{array}$ \\
\hline & $\begin{array}{c}\text { Body } \\
\text { temperature } \\
\text { changes }\end{array}$ & Rct & Serious & No & No & No & No & $\begin{array}{c}\mathrm{MD}=-0.43 \\
(-0.55,-0.31)\end{array}$ & $\begin{array}{l}\oplus \oplus \oplus \oplus \bigcirc \\
\text { moderate }\end{array}$ \\
\hline \multirow{5}{*}{$\begin{array}{l}\text { Xiao et al. } \\
\text { [19] } 2018\end{array}$} & $\begin{array}{l}\text { Duration of } \\
\text { mechanical } \\
\text { ventilation }\end{array}$ & Rct & Serious & No & No & No & No & $\begin{array}{c}\text { SMD }-0.90 \\
(-1.07,-0.72)\end{array}$ & $\begin{array}{l}\oplus \oplus \oplus \oplus \bigcirc \\
\text { moderate }\end{array}$ \\
\hline & $\begin{array}{c}\text { Length of ICU } \\
\text { stay }\end{array}$ & Rct & Serious & No & No & No & No & $\begin{array}{l}\text { SMD }-0.89 \\
(-1.04,-0.73)\end{array}$ & $\begin{array}{l}\oplus \oplus \oplus \oplus \bigcirc \\
\text { moderate }\end{array}$ \\
\hline & $\begin{array}{l}28 \text { days survival } \\
\text { rate }\end{array}$ & Rct & Serious & No & No & No & No & $\begin{array}{c}\text { RR } 1.20 \\
(1.08,1.34)\end{array}$ & $\begin{array}{l}\oplus \oplus \oplus \oplus \bigcirc \\
\text { moderate }\end{array}$ \\
\hline & $\begin{array}{c}\text { Serum levels of } \\
\text { PCT }\end{array}$ & Rct & Serious & Serious & No & No & No & $\begin{array}{l}\text { SMD }-0.57 \\
(-0.77,-0.38)\end{array}$ & $\begin{array}{c}\oplus \oplus \oplus \bigcirc \bigcirc \\
\text { low }\end{array}$ \\
\hline & $\begin{array}{l}\text { APACHE II } \\
\text { score }\end{array}$ & Rct & Serious & Serious & No & No & No & $\begin{array}{l}\text { SMD }-1.16 \\
(-1.57,-0.75)\end{array}$ & $\begin{array}{c}\oplus \oplus \oplus \bigcirc \bigcirc \\
\text { low }\end{array}$ \\
\hline \multirow{4}{*}{$\begin{array}{l}\text { Zheng } \\
\text { et al. [20] } \\
2018\end{array}$} & $\begin{array}{l}28 \text { days } \\
\text { mortality }\end{array}$ & Rct & Serious & No & No & No & No & $\begin{array}{c}\text { RR } 0.64(0.43, \\
0.96)\end{array}$ & $\begin{array}{l}\oplus \oplus \oplus \oplus \bigcirc \\
\text { moderate }\end{array}$ \\
\hline & $\begin{array}{l}\text { APACHE II } \\
\text { score }\end{array}$ & Rct & Serious & Serious & No & No & No & $\begin{array}{c}\text { SMD }-1.21 \\
(-1.62,-0.80)\end{array}$ & $\begin{array}{c}\oplus \oplus \oplus \bigcirc \bigcirc \\
\text { low }\end{array}$ \\
\hline & $\begin{array}{c}\text { Duration of } \\
\text { mechanical } \\
\text { ventilation }\end{array}$ & Rct & Serious & Serious & No & No & No & $\begin{array}{c}\text { SMD }-1.04 \\
(-1.40,-0.67)\end{array}$ & $\begin{array}{c}\oplus \oplus \oplus \bigcirc \bigcirc \\
\text { low }\end{array}$ \\
\hline & $\begin{array}{l}\text { Length of ICU } \\
\text { stay }\end{array}$ & Rct & Serious & No & No & No & No & $\begin{array}{l}\text { SMD }-0.83 \\
(-1.03,-0.64)\end{array}$ & $\begin{array}{l}\oplus \oplus \oplus \oplus \bigcirc \\
\text { moderate }\end{array}$ \\
\hline \multirow{5}{*}{$\begin{array}{l}\text { Xiao et al. } \\
\text { [21] } 2017\end{array}$} & $\begin{array}{l}28 \text { days } \\
\text { mortality }\end{array}$ & Rct & Serious & No & No & No & Serious & $\begin{array}{c}\text { RR } 0.51 \\
(0.44,0.59)\end{array}$ & $\begin{array}{c}\oplus \oplus \oplus \bigcirc \bigcirc \\
\text { low }\end{array}$ \\
\hline & $\begin{array}{l}\text { APACHE II } \\
\text { score }\end{array}$ & Rct & Serious & Serious & No & No & No & $\begin{array}{l}\text { WMD }-3.70 \\
(-4.31,-3.09)\end{array}$ & $\begin{array}{c}\oplus \oplus \oplus \bigcirc \bigcirc \\
\text { low }\end{array}$ \\
\hline & $\begin{array}{c}\text { Serum levels of } \\
\text { PCT }\end{array}$ & Rct & Serious & Serious & No & No & No & $\begin{array}{l}\text { WMD }-1.26 \\
(-1.63,-0.88)\end{array}$ & $\begin{array}{c}\oplus \oplus \oplus \bigcirc \bigcirc \\
\text { low }\end{array}$ \\
\hline & $\begin{array}{l}\text { White blood } \\
\text { count }\end{array}$ & Rct & Serious & Serious & No & No & No & $\begin{array}{l}\text { WMD }-1.48 \\
(-2.03,-0.94)\end{array}$ & $\begin{array}{c}\oplus \oplus \oplus \bigcirc \bigcirc \\
\text { low }\end{array}$ \\
\hline & $\begin{array}{c}\text { Body } \\
\text { temperature } \\
\text { changes }\end{array}$ & Rct & Serious & Serious & No & No & No & $\begin{array}{c}\text { WMD }-0.50 \\
(-0.92,-0.07)\end{array}$ & $\begin{array}{c}\oplus \oplus \oplus \bigcirc \bigcirc \\
\text { low }\end{array}$ \\
\hline \multirow{3}{*}{$\begin{array}{l}\text { Liu et al. } \\
\text { [22] } 2021\end{array}$} & $\begin{array}{l}28 \text { days } \\
\text { mortality }\end{array}$ & Rct & Serious & No & No & No & No & $\begin{array}{c}\text { RR } 1.20 \\
(1.15,1.25)\end{array}$ & $\begin{array}{l}\oplus \oplus \oplus \oplus \bigcirc \\
\text { moderate }\end{array}$ \\
\hline & $\begin{array}{l}\text { White blood } \\
\text { count }\end{array}$ & Rct & Serious & Serious & No & No & No & $\begin{array}{c}\mathrm{MD}-1.95 \\
(-3.62,-0.28)\end{array}$ & $\begin{array}{c}\oplus \oplus \oplus \bigcirc \bigcirc \\
\text { low }\end{array}$ \\
\hline & $\begin{array}{c}\text { Serum levels of } \\
\text { PCT }\end{array}$ & Rct & Serious & Serious & No & No & No & $\begin{array}{c}\mathrm{MD}-1.29 \\
(-1.97,-0.62)\end{array}$ & $\begin{array}{c}\oplus \oplus \oplus \bigcirc \bigcirc \\
\text { low }\end{array}$ \\
\hline
\end{tabular}


TABle 4: Continued.

\begin{tabular}{|c|c|c|c|c|c|c|c|c|c|}
\hline \multirow{2}{*}{ Review } & \multirow{2}{*}{ Outcomes } & \multicolumn{6}{|c|}{ Certainty assessment } & \multirow{2}{*}{$\begin{array}{l}\text { Relative effect } \\
\quad(95 \% \text { CI })\end{array}$} & \multirow{2}{*}{ Quality } \\
\hline & & Design & Limitations & Inconsistency & Indirectness & Imprecision & $\begin{array}{l}\text { Publication } \\
\text { bias }\end{array}$ & & \\
\hline \multirow{2}{*}{$\begin{array}{l}\text { Zhang } \\
\text { et al. [23] } \\
2021\end{array}$} & $\begin{array}{l}28 \text { days } \\
\text { mortality }\end{array}$ & Rct & Serious & No & No & No & No & $\begin{array}{c}\text { OR } 0.52 \\
(0.38,0.71)\end{array}$ & $\begin{array}{l}\oplus \oplus \oplus \oplus \bigcirc \\
\text { moderate }\end{array}$ \\
\hline & $\begin{array}{l}\text { APACHE II } \\
\text { score }\end{array}$ & Rct & Serious & No & No & No & No & $\begin{array}{l}\text { WMD }-2.65 \\
(-3.23,-2.08)\end{array}$ & $\begin{array}{c}\oplus \oplus \oplus \oplus \bigcirc \\
\text { moderate }\end{array}$ \\
\hline $\begin{array}{l}\text { Zhou } \\
\text { et al. [24] } \\
2016 \\
\end{array}$ & $\begin{array}{l}28 \text { days } \\
\text { mortality }\end{array}$ & Rct & Serious & No & No & Serious & No & $\begin{array}{c}\text { RR } 0.61 \\
(0.41,0.90)\end{array}$ & $\begin{array}{c}\oplus \oplus \oplus \bigcirc \bigcirc \\
\text { low }\end{array}$ \\
\hline \multirow{4}{*}{$\begin{array}{l}\text { Li et al. } \\
\text { [25] } 2016\end{array}$} & Effective rate & Rct & Serious & No & No & No & No & $\begin{array}{c}\text { OR } 2.90 \\
(1.89,4.47)\end{array}$ & $\begin{array}{l}\oplus \oplus \oplus \oplus \bigcirc \\
\text { moderate }\end{array}$ \\
\hline & $\begin{array}{l}\text { APACHE II } \\
\text { score }\end{array}$ & Rct & Serious & No & No & No & No & $\begin{array}{c}\mathrm{MD}-4.01 \\
(-4.88,-3.13)\end{array}$ & $\begin{array}{l}\oplus \oplus \oplus \oplus \bigcirc \\
\text { moderate }\end{array}$ \\
\hline & $\begin{array}{l}\text { White blood } \\
\text { count }\end{array}$ & Rct & Serious & No & No & Serious & Serious & $\begin{array}{c}\mathrm{MD}-4.31 \\
(-6.73,-1.89)\end{array}$ & $\begin{array}{l}\oplus \bigcirc \bigcirc \bigcirc \bigcirc \\
\text { very low }\end{array}$ \\
\hline & $\begin{array}{c}\text { Serum levels of } \\
\text { PCT }\end{array}$ & Rct & Serious & No & No & Serious & Serious & $\begin{array}{c}\mathrm{MD}-1.42 \\
(-1.90,-0.95)\end{array}$ & $\begin{array}{l}\oplus 0000 \\
\text { very low }\end{array}$ \\
\hline $\begin{array}{l}\text { Xu et al. } \\
\text { [26] } 2014\end{array}$ & $\begin{array}{l}28 \text { days survival } \\
\text { rate }\end{array}$ & Rct & Serious & No & No & No & No & $\begin{array}{c}\text { RR } 1.21 \\
(1.12,1.29)\end{array}$ & $\begin{array}{l}\oplus \oplus \oplus \oplus \bigcirc \\
\text { moderate }\end{array}$ \\
\hline \multirow{3}{*}{$\begin{array}{l}\text { Li et al. } \\
\text { [27] } 2014\end{array}$} & $\begin{array}{l}28 \text { days } \\
\text { mortality }\end{array}$ & Rct & Serious & No & No & No & No & $\begin{array}{c}\text { OR } 0.39 \\
(0.27,0.58)\end{array}$ & $\begin{array}{l}\oplus \oplus \oplus \oplus \bigcirc \\
\text { moderate }\end{array}$ \\
\hline & $\begin{array}{l}\text { APACHE II } \\
\text { score }\end{array}$ & Rct & Serious & Serious & No & No & No & $\begin{array}{l}\text { WMD }-3.43 \\
(-4.72,-2.15)\end{array}$ & $\begin{array}{l}\oplus \oplus \oplus \bigcirc \bigcirc \\
\text { low }\end{array}$ \\
\hline & $\begin{array}{l}\text { White blood } \\
\text { count }\end{array}$ & Rct & Serious & No & No & Serious & Serious & $\begin{array}{l}\text { WMD }-2.94 \\
(-3.49,-2.38 \\
\end{array}$ & $\begin{array}{l}\oplus \bigcirc \bigcirc \bigcirc \bigcirc \\
\text { very low }\end{array}$ \\
\hline $\begin{array}{l}\text { Sun et al. } \\
\text { [28] } 2012\end{array}$ & $\begin{array}{l}\text { White blood } \\
\text { count }\end{array}$ & Rct & Serious & Serious & No & No & No & $\begin{array}{l}\text { WMD }-1.87 \\
(-2.92,-0.81)\end{array}$ & $\begin{array}{c}\oplus \oplus \oplus \bigcirc \bigcirc \\
\text { low }\end{array}$ \\
\hline $\begin{array}{l}\text { Hu et al. } \\
\text { [29] } 2010\end{array}$ & $\begin{array}{l}28 \text { days } \\
\text { mortality }\end{array}$ & Rct & Serious & Serious & No & No & No & $\begin{array}{c}\text { RR } 0.65 \\
(0.54,0.79) \\
\end{array}$ & $\begin{array}{c}\oplus \oplus \oplus \bigcirc \bigcirc \\
\text { low }\end{array}$ \\
\hline \multirow{4}{*}{$\begin{array}{l}\text { Wu et al. } \\
\text { [30] } 2020\end{array}$} & $\begin{array}{l}28 \text { days } \\
\text { mortality }\end{array}$ & Rct & Serious & No & No & No & No & $\begin{array}{c}\text { RR } 0.52 \\
(0.40,0.67)\end{array}$ & $\begin{array}{l}\oplus \oplus \oplus \oplus \bigcirc \\
\text { moderate }\end{array}$ \\
\hline & $\begin{array}{l}\text { APACHE II } \\
\text { score }\end{array}$ & Rct & Serious & Serious & No & No & No & $\begin{array}{c}\mathrm{MD}-5.48 \\
(-7.52,-3.43)\end{array}$ & $\begin{array}{l}\oplus \oplus \oplus \bigcirc \bigcirc \\
\text { low }\end{array}$ \\
\hline & $\begin{array}{l}\text { White blood } \\
\text { count }\end{array}$ & Rct & Serious & Serious & No & No & No & $\begin{array}{c}\mathrm{MD}-2.26 \\
(-3.35,-1.17)\end{array}$ & $\begin{array}{c}\oplus \oplus \oplus \bigcirc \bigcirc \\
\text { low }\end{array}$ \\
\hline & $\begin{array}{l}\text { C-reactive } \\
\text { protein }\end{array}$ & Rct & Serious & Serious & No & No & No & $\begin{array}{c}\mathrm{MD}-37.43 \\
(-56.70 \\
-18.16)\end{array}$ & $\begin{array}{c}\oplus \oplus \oplus \bigcirc \bigcirc \\
\text { low }\end{array}$ \\
\hline
\end{tabular}

ICU, intensive care unit; APACHE, acute physiology and chronic health evaluation; PCT, procalcitonin. RCT, randomized controlled trials; WMD, weighted mean difference; SMD, standard mean difference; $\mathrm{MD}$, mean difference; OR, odds ratio; RR, relative risk.

Based on the results of GRADE, only three outcome indicators provided high-quality evidence, 16 provided moderate-quality evidence, and the remaining 24 provided low or critical low-quality evidence. These results indicate that the conclusions of the reviews may differ from the true results and therefore cannot be used as an evidence-based basis. Furthermore, it is worth noting that almost all the included SRs/MAs indicated that XBJI plus WM seems to have significant clinical efficacy in the therapy of patients with sepsis. However, most authors did not wish to draw definitive conclusions due to low methodological quality or the small size of the enrolled studies.

The pathogenesis of sepsis includes inflammation, immune dysregulation, and coagulopathy, with uncontrolled inflammation being the most critical for patients [31]. According to traditional Chinese medicine, the basic pathogenesis of sepsis involves the accumulation of toxins in the interior and extremities, leading to siltation, stagnation, and weakened body resistance [10]. XBJI was then created according to this theory as a possible treatment for sepsis [32]. XBJI was composed of five herbs containing approximately 30 bioactive compounds, including hydroxysafflor yellow A, danshenol, ferulic acid, paeoniflorin, and senna lactone I, among others [17]. Therefore, XBJI has the effects of "multiingredient, multitarget, and multipathway," including detoxifying and toning, elimination of bacteria and viruses, supplementing vital energy, and invigorating blood circulation [19]. Modern pharmacological studies have uncovered the potential therapeutic mechanisms of XBJI for sepsis. It was reported that XBJI could regulate the immune status of the body by inhibiting the release of inflammatory mediators, reducing the total accumulation of endotoxins, 
bacterial toxin detoxification, and reducing the total amount of oxygen free radicals in the circulatory system. These effects help regulate the overall microcirculatory status of the body, protect and restore vascular endothelial function, and increase the total blood perfusion of the organs [19]. Moreover, XBJI also reduces the release of mast cells, which reduces the synthetic activity of fibroblasts. These effects lead to the avoidance of inflammatory exudation and increase the permeability of blood vessels [11]. Furthermore, XBJI helps the body absorb necrotic material and hematoma, promoting rehabilitation [33]. Thus, the use of XBJI is considered a promising approach for the treatment of sepsis.

To our knowledge, this is the first study to systematically collate, appraise, and synthesize the scientific evidence on XBJI for sepsis. However, we found that most of the included reviews were of poor quality, which could result in these studies having low credibility. Furthermore, the AMSTAR-2 tool, PRISMA checklist, and the GRADE system are highly subjective as different reviewers have their independent judgment. The subjectivity of the authors may then lead to varying results as subjective factors or errors cannot be eliminated.

\section{Conclusion}

The combination of XBJI and WM showed significant synergy for the treatment of sepsis compared to the use of WM alone. It provided a new and prospective therapeutic method for sepsis. However, this conclusion should be treated with caution as the quality of SRs/MAs providing evidence was generally low.

\section{Abbreviations}

$\begin{array}{ll}\text { XBJI: } & \text { Xuebijing injection } \\ \text { WM: } & \text { Western medicines } \\ \text { SR: } & \text { Systematic review } \\ \text { MA: } & \text { Meta-analysis } \\ \text { AMSTAR- } & \text { Assessing the Methodological Quality of } \\ \text { 2: } & \text { Systematic Reviews-2 } \\ \text { GRADE: } & \text { Grading of Recommendations, Assessment, } \\ & \text { Development, and Evaluation } \\ \text { PRISMA: } & \text { Preferred Reporting Item for Systematic } \\ & \text { Reviews and Meta-Analyses } \\ \text { ICU: } & \text { Intensive care unit } \\ \text { APACHE: } & \text { Acute physiology and chronic health evaluation } \\ \text { PCT: } & \text { Procalcitonin } \\ \text { RCT: } & \text { Randomized controlled trials } \\ \text { WMD: } & \text { Weighted mean difference } \\ \text { SMD: } & \text { Standard mean difference } \\ \text { MD: } & \text { Mean difference } \\ \text { OR: } & \text { Odds ratio } \\ \text { RR: } & \text { Relative risk. }\end{array}$

\section{Data Availability}

The data generated or analyzed during this study are included within this article.

\section{Conflicts of Interest}

The authors declare that they have no conflicts of interest.

\section{Authors' Contributions}

Sa Tian designed and drafted the study. Yixuan Ye, Defang Qin, Huawei Yang, and Shuguang Chen performed the literature search, literature selection, and data extraction. Tao Liu, Luming Hu, Huiming Li, and Qin Niu performed the quality assessment. All authors approved the final version of the manuscript.

\section{References}

[1] M. Singer, C. S. Deutschman, C. W. Seymour et al., "The third international consensus definitions for sepsis and septic shock (Sepsis-3)," Journal of the American Medical Association, vol. 315 , no. 8, pp. 801-810, 2016.

[2] K. M. Kaukonen, M. Bailey, S. Suzuki, D. Pilcher, and R. Bellomo, "Mortality related to severe sepsis and septic shock among critically ill patients in Australia and New Zealand, 2000-2012," JAMA, vol. 311, no. 13, pp. 1308-1316, 2014.

[3] V. Liu, G. J. Escobar, J. D. Greene et al., "Hospital deaths in patients with sepsis from 2 independent cohorts," Journal of the American Medical Association, vol. 312, no. 1, pp. 90-92, 2014.

[4] C. Fleischmann, A. Scherag, N. K. J. Adhikari et al., "Assessment of global incidence and mortality of hospital-treated sepsis. Current estimates and limitations," American Journal of Respiratory and Critical Care Medicine, vol. 193, no. 3, pp. 259-272, 2016.

[5] V. Y. Dombrovskiy, A. A. Martin, J. Sunderram, and H. L. Paz, "Rapid increase in hospitalization and mortality rates for severe sepsis in the United States: a trend analysis from 1993 to 2003*," Critical Care Medicine, vol. 35, no. 5, pp. 1244-1250, 2007.

[6] R. P. Dellinger, M. Levy Mitchell, R. Andrew et al., "Surviving Sepsis Campaign: international guidelines for management of severe sepsis and septic shock, 2012," Intensive Care Medicine, vol. 39, no. 2, pp. 165-228, 2013.

[7] X. Y. Sun, X. F. Ding, H. Y. Liang et al., "Efficacy of mesenchymal stem cell therapy for sepsis: a meta-analysis of preclinical studies," Stem Cell Research \& Therapy, vol. 11, no. 1, p. 214, 2020.

[8] C. Li, P. Wang, M. Li et al., "The current evidence for the treatment of sepsis with Xuebijing injection: bioactive constituents, findings of clinical studies and potential mechanisms," Journal of Ethnopharmacology, vol. 265, p. 113301, 2021.

[9] S. T. Ma, P. X. Liu, W. Long, J. Yu, and Y. Xu, "Effects of the multi-target capability of Xuebijing and its inflammatory pharmacodynamic material basis," Acta Physico-Chimica Sinica, vol. 15, no. 10, pp. 2080-2086, 2009.

[10] Q. Yin and C. S. Li, "Treatment effects of Xuebijing injection in severe septic patients with disseminated intravascular coagulation," Evidence Based Complementary and Alternative Medicine, vol. 2014, Article ID 949254, 2014.

[11] Y. Y. Feng, Y. Y. Xie, Y. P. Wang et al., "Molecular mechanism of Xuebijing injection in treatment of sepsis according to "drug-target-pathway" network," Acta Physico-Chimica Sinica, vol. 52, no. 4, pp. 556-562, 2017. 
[12] J. K. Huang, X. H. Qin, M. Shen, and Y. Huang, “An overview of systematic reviews and meta-analyses on acupuncture for post-stroke aphasia," European Journal of Integrative Medicine, vol. 37, 2020.

[13] J. Huang, Y. Wang, S. Huang et al., "A critical overview of systematic reviews of shenfu injection for heart failure," Cardiovascular Therapeutics, vol. 2021, no. 16, 9 pages, 2021.

[14] B. J. Shea, B. C. Reeves, G. Wells et al., "AMSTAR 2: a critical appraisal tool for systematic reviews that include randomized or non-randomised studies of healthcare interventions, or both," BMJ, vol. 358, no. j4008, p. 1, 2017.

[15] A. Liberati, D. G. Altman, J. Tetzlaff et al., "The PRISMA statement for reporting systematic reviews and meta-analyses of studies that evaluate healthcare interventions: explanation and elaboration," BMJ, vol. 339, no. jul21 1, p. b2700, 2009.

[16] G. H. Guyatt, A. D. Oxman, G. E. Vist et al., "GRADE: an emerging consensus on rating quality of evidence and strength of recommendations," BMJ, vol. 336, no. 7650, pp. 924-926, 2008.

[17] G. Chen, Y. Gao, Y. Jiang et al., "Efficacy and safety of Xuebijing injection combined with ulinastatin as adjunctive therapy on sepsis: a systematic review and meta-analysis," Frontiers in Pharmacology, vol. 9, p. 743, 2018.

[18] C. Li, P. Wang, L. Zhang et al., "Efficacy and safety of Xuebijing injection (a Chinese patent) for sepsis: a metaanalysis of randomized controlled trials," Journal of Ethnopharmacology, vol. 224, pp. 512-521, 2018.

[19] S.-H. Xiao, L. Luo, X.-H. Liu, Y.-M. Zhou, H.-M. Liu, and Z.-F. Huang, "Curative efficacy and safety of traditional Chinese medicine Xuebijing injections combined with ulinastatin for treating sepsis in the Chinese population," Medicine (Baltimore), vol. 97, no. 23, p. e10971, 2018.

[20] J. Zheng, X. Xiang, B. Xiao et al., "Xuebijing combined with ulinastation benefits patients with sepsis: a meta-analysis," The American Journal of Emergency Medicine, vol. 36, no. 3, pp. 480-487, 2018.

[21] H. Shi, Y. Hong, J. Qian, X. Cai, and S. Chen, "Xuebijing in the treatment of patients with sepsis," The American Journal of Emergency Medicine, vol. 35, no. 2, pp. 285-291, 2017.

[22] J. T. Liu, X. D. Cao, and H. F. Li, "Meta-analysis of the effectiveness of Xuebijing injection in the treatment of sepsis," Journal of Shanxi University of Chinese Medicine, vol. 22, no. 1, pp. 12-18, 2021.

[23] J. W. Zhang, Y. L. He, Y. Shi, and M. Li, "Meta-analysis of the efficacy and safety of Xuebijing injection in the treatment of vascular endothelial injury in sepsis," Journal of Hainan Medical University, vol. 27, no. 12, pp. 910-917, 2020.

[24] X. S. Zhou, G. H. Tang, J. Y. Li, and Y. Ye, "Xuebijing injection reducing mortality of septic shock patients: a systematic review and meta-analysis," Chinese Archives of Traditional Chinese Medicine, vol. 34, no. 9, pp. 2161-2164, 2016.

[25] G. Li, Y. Xiao, J. Zhu, X. Ma, and W. Gao, "Systematic review of Xuebijing injection in treatment of septic shock," Journal of Emergency in Traditional Chinese Medicine, vol. 25, no. 5, pp. 834-838, 2016.

[26] Y. Xu, Y. Geng, H. Tong, H. Li, P. Wan, and L. Su, "Metaanalysis of Xuebijing injection in improving survival rate of patients with sepsis," Clinical Journal of Traditional Chinese Medicine, vol. 26, no. 5, pp. 456-460, 2014.

[27] N. Li, W. Jiang, L. Yu, and W. Zhang, "Systematic review of Xuebijing injection in treatment of sepsis," Chinese Journal of Modern Drug Application, vol. 7, no. 22, pp. 8-11, 2013.

[28] C. L. Sun, Y. Zhuang, and X. Wang, "Meta-analysis of Xuebijing injection in the treatment of sepsis," Journal of
Emergency in Traditional Chinese Medicine, vol. 21, no. 3, pp. 411-413, 2012.

[29] J. Hu, H. Shang, J. Li, J. Li, Z. Zhang, and B. Zhang, "Injection for sepsis: a comprehensive review," Medical Journal of Chinese People's Liberation Army, vol. 35, no. 1, pp. 9-12, 2010.

[30] Y. J. Wu, J. Zhang, and L. J. Qi, "Clinical efficacy and safety of Xuebijing injection on sepsis: a meta-analysis," Chinese Critical Care Medicine, vol. 32, no. 6, pp. 691-695, 2020.

[31] K. Kubra, H. Beatrice, E. C. Steve et al., "Human metabolic response to systemic inflammation: assessment of the concordance between experimental endotoxemia and clinical cases of sepsis/SIRS," Critical Care, vol. 19, p. 71, 2015.

[32] J. D. Wang, "Diagnosis and treatment ideas and practice course of integrative treatment in acute critical illness," Tianjin Journal of Traditional Chinese Medicine, vol. 15, pp. 241-242, 1998.

[33] W. Zhang, Y. N. Zhu, and Y. Wang, "Application of Xuebijing injection in clinical application of infectious diseases," Lishizhen Medicine and Materia Medica Research, vol. 25, no. 5, pp. 1189-1191, 2014. 